13

\title{
Пробеги атомов водорода, дейтерия, гелия в аморфных кремнии и вольфраме
}

\author{
(ㄱ Д.С. Мелузова, П.Ю. Бабенко, А.П. Шергин, А.Н. Зиновьев
}

Физико-технический институт им. А.Ф. Иофрфе РАН,

194021 Санкт-Петербург, Россия

e-mail: dmeluzova@gmail.com

Поступило в Редакцию 9 марта 2019 г.

В окончательной редакции 7 июня 2019 г.

Принято к публикации 29 июня 2019 г.

Получены распределения пробегов для атомов $\mathrm{H}, \mathrm{D}, \mathrm{He}$ с энергиями в диапазоне $0.1-100 \mathrm{keV}$ в аморфных W и $\mathrm{Si}$ для нормального падения налетающих частиц. Расчеты проведены как в приближении парных соударений, так и более точным методом расчета траекторий частиц. Показано, что результаты обоих методов хорошо согласуются между собой и с экспериментальными данными по средним пробегам для системы $\mathrm{H}-\mathrm{Si}$. Оценено влияние притягивающей ямы в потенциале взаимодействия налетающей частицы с атомами твердого тела на результаты расчетов.

Ключевые слова: пробеги, вольфрам, кремний, аморфный.

DOI: $10.21883 / J T F .2020 .01 .48678 .89-19$

\section{Введение}

Одной из серьезных практических проблем при создании токамака-реактора в области физики плазмы является проблема радиационных повреждений материалов при взаимодействии атомов плазмы с вольфрамом материалом дивертора токамака-реактора. Достоверные данные о пробегах атомных частиц в аморфном вольфраме отсутствуют. Данные о пробегах частиц нужны для оценок создания дефектов и накопления изотопов водорода в материале.

Различают полный пробег частицы, т.е. длину пути частицы, и проективный пробег - проекцию пробега на выбранное направление (обычно перпендикулярно поверхности). Вследствие многократности соударений имеет место распределение проективного пробега по глубине. В настоящей работе под пробегом мы понимаем среднее значение проективного пробега.

Значительный вклад в развитие моделирования рассеяния частиц на поверхности внесли отечественные авторы [1-3]. Наиболее подробно различные модели описаны в монографии [4].

Широко используется код SRIM [5], основанный на приближении парных соударений, которое было предложено в работе [6]. К недостаткам кода SRIM можно отнести невозможность свободно менять рассеивающий потенциал, структуру мишени, модель описания неупругих потерь энергии.

Нами был разработан численный код для моделирования рассеяния атомных частиц на поверхности аморфных, кристаллических и поликристаллических мишеней $[7,8]$. Благодаря гибкости данного кода была осуществлена его модернизация с целью использования для определения глубины проникновения атомных частиц в различные материалы.

\section{1. Методика расчета}

Как показывают рентгеновские исследования аморфных тел, в положении атомов в аморфном теле наблюдается ближний порядок. Чтобы учесть это явление, мы строили случайно ориентированный в пространстве плотно упакованный кластер с расстоянием между атомами $d$, определяемым из плотности мишени. Поверхность определяется как случайный срез данного кластера.

В настоящей работе расчеты проводились с помощью приближения парных соударений (Binary Collision Approximation - BCA) и методом расчета траекторий.

В приближении парных соударений рассеяние атомных частиц в твердом теле рассматривается как последовательность парных соударений атомов. Траектория движения частицы заменяется асимптотами траектории. Подобный подход позволяет существенно сократить время счета. При энергии соударения ниже $50 \mathrm{eV}$ применимость приближения нарушается.

Угол рассеяния атомной частицы определяется известным выражением

$$
\theta=\pi-2 p \int_{r_{0}}^{\infty} \frac{d r / r^{2}}{\sqrt{1-U(r) / E-p^{2} / r^{2}}}
$$

где $r$ - расстояние между частицами, $U(r)-$ потенциал взаимодействия, $p$ - прицельный параметр, $E$ - энергия налетающей частицы, $r_{0}-$ расстояние наибольшего сближения, являющееся корнем уравнения $1-U\left(r_{0}\right) / E-p^{2} / r_{0}^{2}=0$. Заменой $r=r_{0} / \cos x$ устраняется особенность в знаменателе подынтегрального 
выражения и формула (1) преобразуется к виду

$$
\theta=\pi-2 \int_{0}^{\pi / 2} \frac{p / r_{0} \sin x d x}{\sqrt{1-(U(x)) / E-p^{2}\left(\cos x / r_{0}\right)^{2}}} .
$$

Проводится численное интегрирование с использованием разложения по полиномам Гаусса с количеством узлов до 20 и точностью $10^{-8}$ от значения интеграла. Как правило, потенциал взаимодействия известен с точностью $15 \%$, что и определяет точность расчета.

Пересчет энергии и координаты частицы после соударения производится по формулам из работы [6]. Значения электронных тормозных способностей $d E / d x$ брались из работы [9]. Ядерные тормозные способности зависят от используемого потенциала, и в наших расчетах они учитывались непосредственно при рассмотрении кинематики конкретного столкновения, а угол рассеяния рассчитывался с использованием выбранного потенциала.

При следующем акте рассеяния мы производили трансляцию (перенос) кластера в пространстве, снова разыгрывая его ориентацию случайным образом. Далее велся перебор по всем атомам кластера в полусфере по направлению движения частицы, и определялось значение параметра удара для каждого рассматриваемого атома из кластера. Очередной рассеивающий атом выбирался из критерия минимума параметра удара.

При рассеянии над поверхностью учитывается отсутствие атомов в верхней полусфере. Счет заканчивается, если энергия атома достигла значения $2 \mathrm{eV}$ или атом отлетел от поверхности на расстояние, большее $3 d$.

При использовании метода расчета траекторий рассматривается взаимодействие налетающей частицы со всеми соседними атомами, что позволяет учитывать многочастичные эффекты, но повышает время счета.

Данный метод состоит в решении уравнений движения частицы:

$$
\begin{gathered}
d \mathbf{x}_{0} / d t=\mathbf{v} \\
\frac{d \mathbf{v}}{d t}=\frac{1}{m} \mathbf{F}=\frac{1}{m} \sum_{j} \mathbf{F}_{j}\left(\left|\mathbf{x}_{0}-\mathbf{x}_{j}\right|\right) \frac{\left(\mathbf{x}_{0}-\mathbf{x}_{j}\right)}{\left(\left|\mathbf{x}_{0}-\mathbf{x}_{j}\right|\right)},
\end{gathered}
$$

где $\mathbf{x}_{0}$ и $\mathbf{v}$ - векторы, описывающие положение и скорость налетающей частицы, $\mathbf{x}_{j}-$ координаты частиц мишени (в данном случае атомов базового кластера радиуса $d), m$ - масса налетающей частицы. Сила $\mathbf{F}_{j}$ определяется, как градиент потенциала взаимодействия между налетающей частицей и атомами мишени, и она направлена вдоль вектора $\mathbf{x}_{0}-\mathbf{x}_{j}$. Для каждого атома мишени определяются проекции силы $\mathbf{F}_{j}$ по трем осям координат с помощью умножения на второй множитель в сумме в формуле (2), а затем происходит суммирование по всем атомам мишени для получения результирующей силы $\mathbf{F}$.
Для численного интегрирования был выбран метод Верле [10] в следующем виде:

$$
\begin{aligned}
& \mathbf{x}_{n+1}=\mathbf{x}_{n}+\mathbf{v}_{n} \Delta t+\mathbf{F}_{n}(\Delta t)^{2} /(2 m), \\
& \mathbf{v}_{n+1}=\mathbf{v}_{n}+\left(\mathbf{F}_{n+1}+\mathbf{F}_{n}\right) \Delta t /(2 m) .
\end{aligned}
$$

Здесь $n$ - номер итерации. Шаг по времени выбирался из критерия $\Delta t=0.05 r_{\min } / v$, где $r_{\min }$ - расстояние от налетающего атома до ближайшего атома мишени.

Изменение кинетической энергии частицы определялось по формуле

$$
E_{n+1}=E_{n}-\frac{d E}{d x}\left|x_{n+1}-x_{n}\right|-Q,
$$

где $d E / d x$ - электронные тормозные способности. При расчете методом траекторий необходимо учесть упругие потери энергии. Величина упругих потерь энергии $Q$ определялась при выходе частицы за пределы рассматриваемого кластера. При этом

$$
Q=2 E_{n} \frac{m_{1} m_{2}}{\left(m_{1}+m_{2}\right)^{2}}\left[1-\cos \left(\theta_{\mathrm{cm}}\right)\right]
$$

где $m_{1}, m_{2}$ - массы налетающей частицы и атома мишени, $\theta_{\mathrm{cm}}$ - угол рассеяния в системе центра масс.

В обоих методах расчета учитывались тепловые колебания атомов решетки, при этом сдвиг атомов решетки, вызванный тепловыми колебаниями, описывался распределением Гаусса. Число обрабатываемых траекторий для получения достаточной статистики достигало $10^{6}$. Для каждой траектории фиксировалось расстояние от поверхности, на которое продвинулась частица при своем движении в твердом теле.

\section{2. Средний пробег и параметры, характеризующие распределение пробегов}

Рассмотрим следующую геометрию: поверхность твердого тела задана плоскостью $(y, z)$, ось $x$ направлена

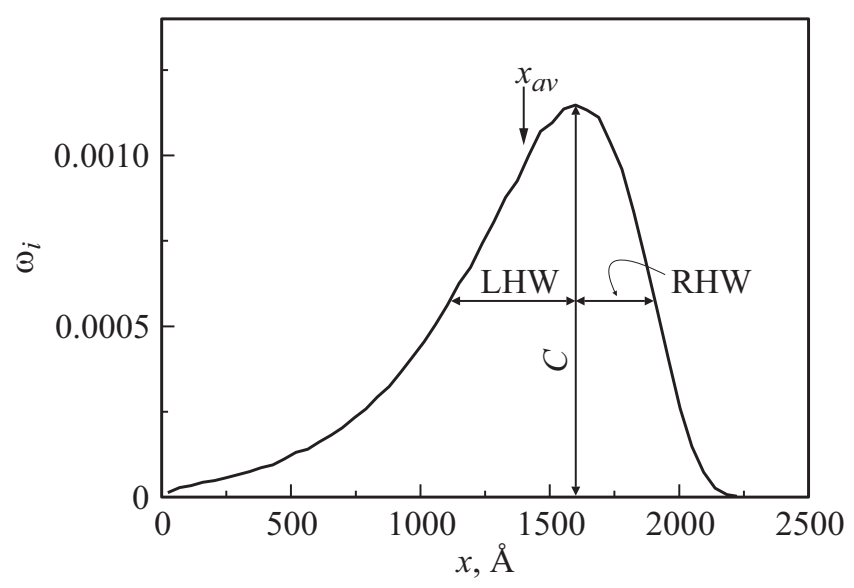

Рис. 1. Распределение пробегов для системы $\mathrm{H}-\mathrm{Si}$, $E=10 \mathrm{keV} ; C$ - положение пика, RHW, LHW - правая и левая полуширина на полувысоте соответственно. 
вглубь мишени, а налетающая частица движется перпендикулярно к поверхности. Пусть налетающая частица остановилась в интервале с координатами $\left[x_{i}, x_{i}+d x\right]$. Распределение пробегов описывается функцией

$$
\omega_{i}=N_{i} / N_{0},
$$

где $N_{0}$ - полное число имплантированных частиц, $N_{i}-$ число частиц с пробегом в рассматриваемом интервале с индексом $i$.

Для описания распределения пробегов используем понятие „средний пробег“, который определяется как среднее значение распределения пробегов следующим образом: $x_{a v}=\Sigma_{i} x_{i} \omega_{i}$.

Для дальнейшего описания распределений мы используем такие величины как положение пика C, правая и левая полуширина на полувысоте (RHW и LHW соответственно). Данные величины изображены на рис. 1.

\section{3. Результаты расчетов}

\section{1. Расчет пробегов для систем $\mathrm{H}-\mathrm{Si}$ и $\mathrm{D}-\mathrm{W}$}

В случае мишени из вольфрама экспериментальные данные и расчеты пробегов отсутствуют. С целью проверки применимости данного кода для расчета пробегов атомных частиц были проведены расчеты для мишени из аморфного кремния, так как в литературе существует большое количество экспериментальных и расчетных данных для данного материала $[4,11,12]$.

На рис. 2 представлены результаты расчетов средних пробегов в зависимости от энергии налетающей частицы в случае бомбардировки аморфного Si атомами Н, а также табличные значения средних пробегов из SRIM и экспериментальные данные $[11,12]$. Расчеты были проведены методом траекторий и методом BCA.

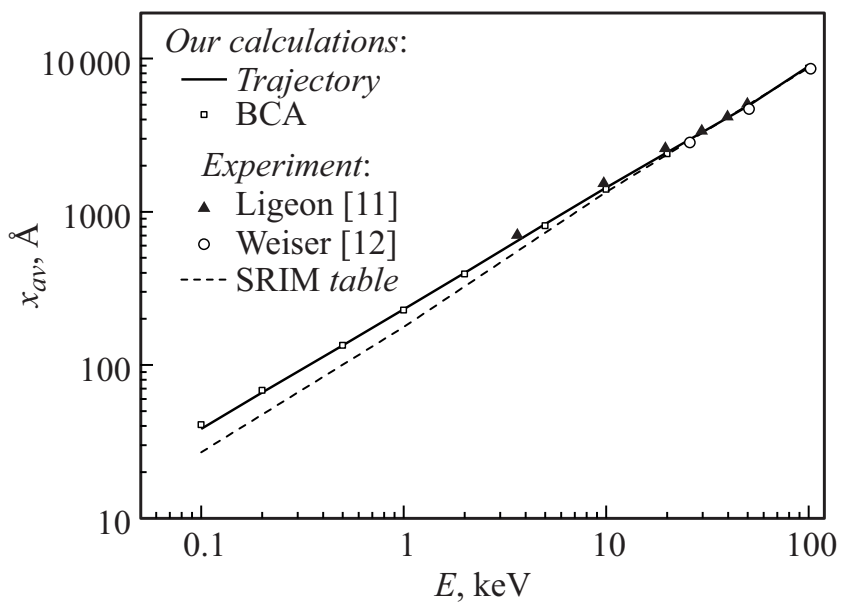

Рис. 2. Зависимость среднего пробега от энергии налетающих частиц для системы H-Si. Расчет методом траекторий (сплошная линия) и BCA (квадраты), табличные значения из SRIM (штриховая линия), экспериментальные данные: треугольники - [11], кружки - [12].

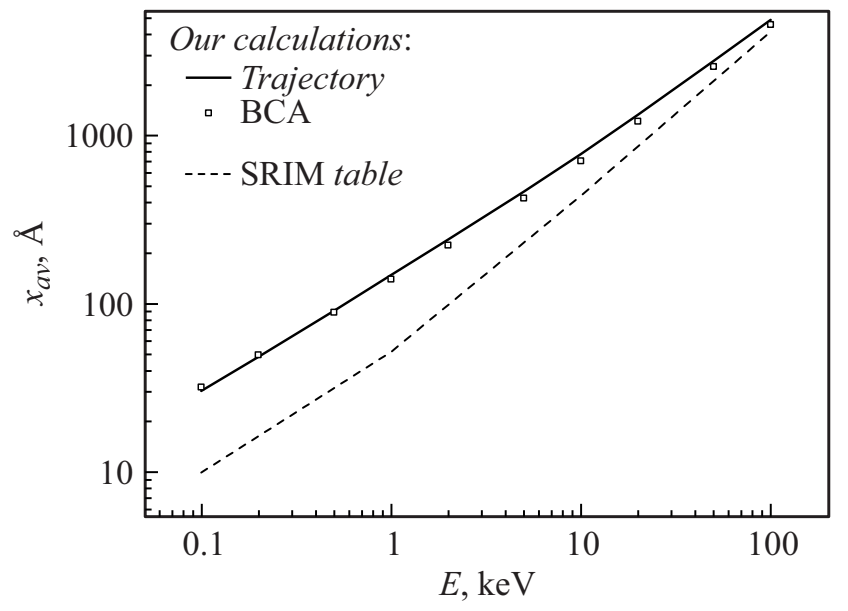

Рис. 3. Зависимость среднего пробега от энергии налетающих частиц для системы D-W. Расчет методом траекторий (сплошная линия) и ВСА (квадраты), табличные значения из SRIM (штриховая линия).

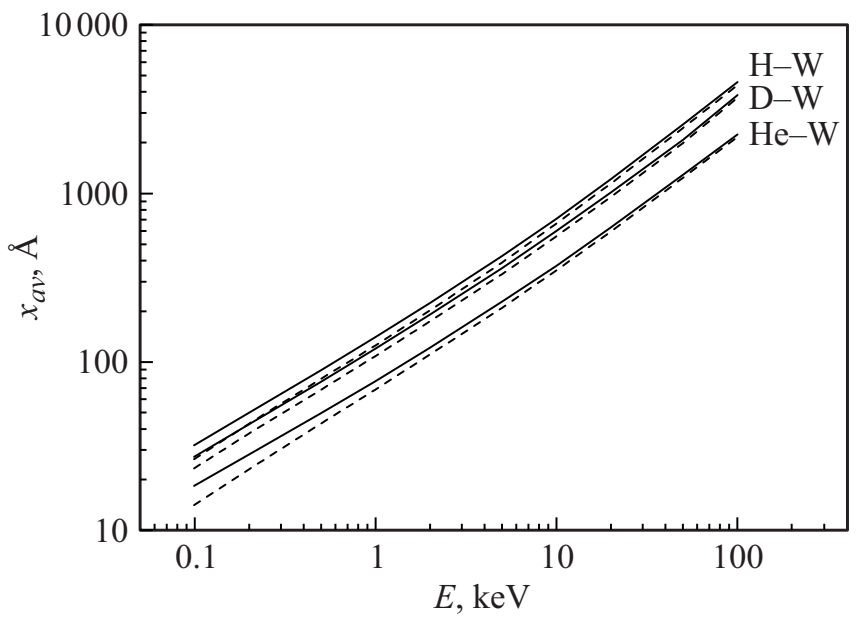

Рис. 4. Сравнение расчетных зависимостей среднего пробега от энергии налетающих частиц для разных потенциалов взаимодействия в системах $\mathrm{H}-\mathrm{W}, \mathrm{D}-\mathrm{W}, \mathrm{He}-\mathrm{W}$, сплошные линии - DFT потенциал, штриховые линии - потенциал ZBL.

В обоих случаях использовался составной потенциал: отталкивающая часть представляет собой потенциал [13], к которому добавлена потенциальная яма с глубиной $3.195 \mathrm{eV}$ и положением минимума $1.5 \AA$ в соответствии со спектроскопическими данными [14]. Оба расчета хорошо согласуются между собой. Также можно видеть хорошее согласие расчетов с экспериментальными данными, особенно в области энергий $\geq 20 \mathrm{keV}$. Полученные результаты для пробегов хорошо описываются формулой

$$
x_{a v}=234 \cdot E^{0.775},
$$

при этом $x_{a v}$ выражено в ангстремах, энергия налетающих частиц $E$ в килоэлектронвольтах.

На рис. 3 представлены результаты расчетов средних пробегов в зависимости от энергии налетающей частицы 
в случае бомбардировки аморфного W атомами D, a также табличные значения средних пробегов из SRIM. Расчеты были проведены методом траекторий и методом ВСА с использованием потенциала, полученного в результате применения метода функционала плотности (DFT) [15]. Для данной системы также наблюдается согласие наших расчетов, проведенных двумя разными методами. Результаты, полученные с использованием DFT-потенциала, могут быть описаны формулой

$$
x_{a v}=144 \cdot E^{0.666}(1+0.00575 \cdot E),
$$

при этом $x_{a v}$ выражено в ангстремах, энергия налетающих частиц $E$ в килоэлектронвольтах.

В случае вольфрама наблюдается значительное различие данных, получаемых при расчете средних пробегов атомов в веществе, с таблицами пробегов, приведенных в SRIM. Различие, хотя и менее значительное, присутствует также для хорошо изученной системы $\mathrm{H}-\mathrm{Si}$. На наш взгляд, таблицы пробегов, приведенные в базе данных SRIM для исследованных нами случаев, нуждаются в серьезной коррекции.

Проведенное сопоставление результатов расчета методом траекторий и методом ВСА показало хорошее согласие данных, получаемых обоими методами. Для расчетов пробегов мы рекомендуем использовать код, построенный на приближении парных соударений, из-за его очевидного преимущества в скорости счета.

\section{2. Влияние потенциала}

Расчеты средних пробегов для атомов $\mathrm{H}, \mathrm{D}, \mathrm{He}$ в аморфном W проводились методом ВСА с использованием потенциалов ZBL (Ziegler-Biersack-Littmark) [16], Зиновьева [13] и потенциала DFT [15]. Отличие потенциала DFT состоит в присутствии притягивающей ямы, в то время как первые два потенциала носят исключительно отталкивательный характер. Значения,

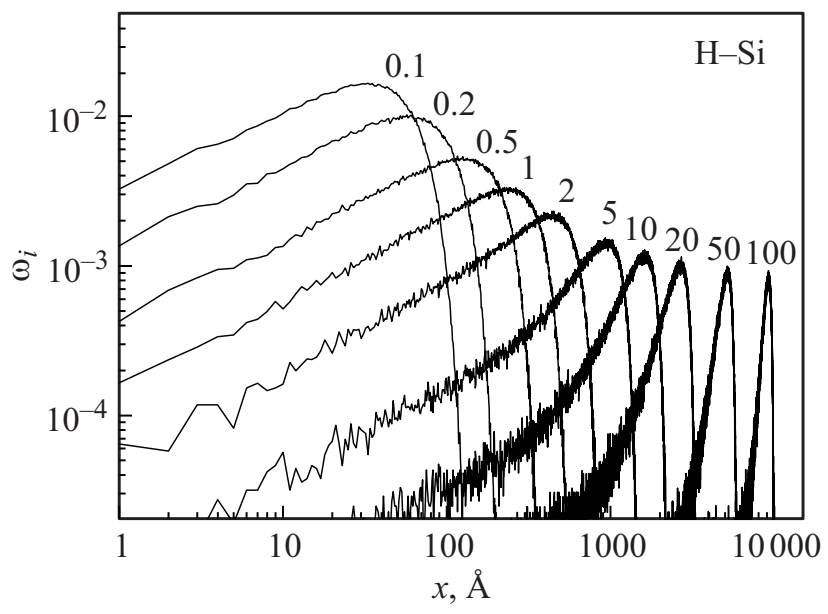

Рис. 5. Распределения пробегов для системы $\mathrm{H}-\mathrm{Si}$ при разных значениях энергии налетающих частиц (цифры у кривых в килоэлектронвольтах).

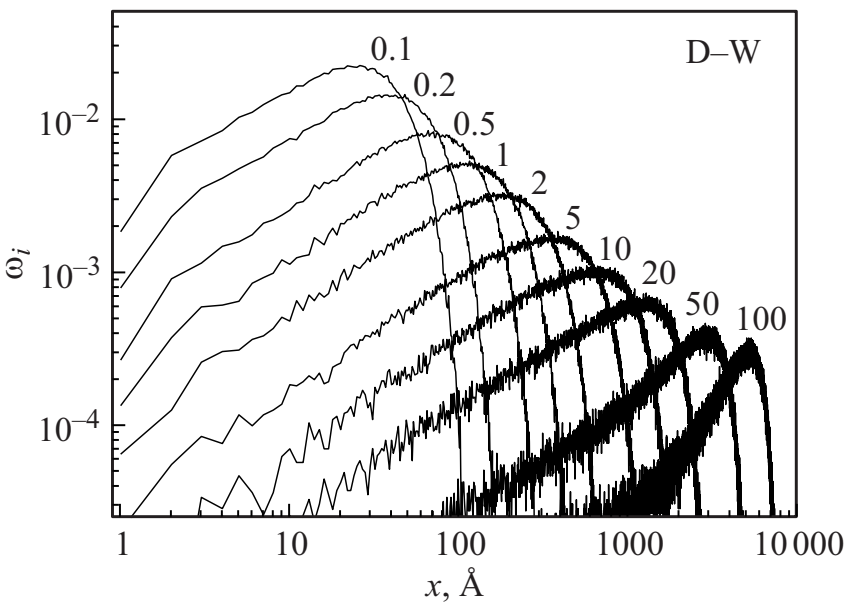

Рис. 6. Распределения пробегов для системы D-W при разных значениях энергии налетающих частиц (цифры у кривых в килоэлектронвольтах).

Таблица 1. Система $\mathrm{H}-\mathrm{Si}$

\begin{tabular}{c|c|c|c|c}
\hline$E, \mathrm{keV}$ & $x_{a v}, \AA$ & $C, \AA$ & LHW, $\AA$ & RHW, $\AA$ \\
\hline 0.1 & 40.8 & 33 & 26.3 & 34.5 \\
0.2 & 68.3 & 58 & 45.8 & 54.8 \\
0.5 & 134.6 & 125 & 98.2 & 99.5 \\
1 & 227.9 & 238 & 179 & 143 \\
2 & 391.5 & 438 & 279 & 193 \\
5 & 809.5 & 938 & 406 & 262 \\
10 & 1399 & 1599 & 487 & 308 \\
20 & 2386 & 2678 & 572 & 318 \\
50 & 4801 & 5153 & 616 & 362 \\
100 & 8626 & 9170 & 640 & 376
\end{tabular}

Примечание: Параметры распределений: $x_{a v}-$ средний пробег, $C-$ положение пика, LHW и RHW - левая и правая полуширина пика на полувысоте.

Таблица 2. Система D-W

\begin{tabular}{c|c|c|c|c}
\hline$E, \mathrm{keV}$ & $x_{a v}, \AA$ & $C, \AA$ & LHW, $\AA$ & RHW, $\AA$ \\
\hline 0.1 & 32.2 & 26.5 & 20.5 & 24.0 \\
0.2 & 50.0 & 39.5 & 29.8 & 39.4 \\
0.5 & 89.5 & 68 & 49.8 & 72.0 \\
1 & 141 & 113 & 85.0 & 114 \\
2 & 224 & 179 & 136 & 181 \\
5 & 425 & 363 & 279 & 333 \\
10 & 708 & 635 & 484 & 527 \\
20 & 1218 & 1324 & 957 & 724 \\
50 & 2572 & 2920 & 1546 & 1051 \\
100 & 4575 & 5255 & 1899 & 1214
\end{tabular}

Примечание: Параметры распределений: $x_{a v}-$ средний пробег, $C-$ положение пика, LHW и RHW - левая и правая полуширина пика на полувысоте.

полученные с использованием потенциалов Зиновьева и ZBL, практически совпадают. Пробеги, полученные с использованием DFT-потенциала, превышают пробеги 


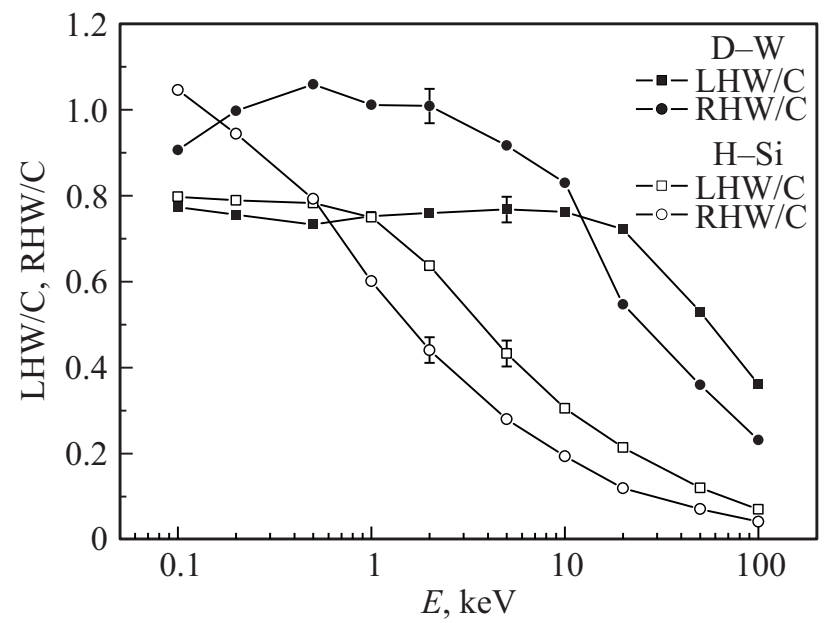

Pис. 7. Зависимость отношений LHW/C и RHW/C от энергии налетающих частиц для систем $\mathrm{H}-\mathrm{Si}$ и $\mathrm{D}-\mathrm{W}$.

для потенциала ZBL. При энергии $100 \mathrm{eV}$ различие составляет 14, 17 и 23\% для систем $\mathrm{H}-\mathrm{W}, \mathrm{D}-\mathrm{W}$ и $\mathrm{He}-\mathrm{W}$ соответственно. Как видно из рис. 4, с ростом энергии влияние выбора потенциала ослабевает. Это можно объяснить тем, что от потенциала зависят величины ядерных потерь энергии, но они на несколько порядков меньше электронных потерь, которые вносят основной вклад при торможении частиц в твердом теле.

Результаты, полученные с использованием DFT-потенциала, могут быть описаны для случая $\mathrm{H}-\mathrm{W}$ формулой

$$
x_{a v}=122 \cdot E^{0.662}(1+0.00509 \cdot E)
$$

для случая $\mathrm{He}-\mathrm{W}$

$$
x_{a v}=79.3 \cdot E^{0.649}(1+0.00468 \cdot E),
$$

при этом $x_{a v}$ выражено в ангстремах, энергия налетающих частиц $E$ в килоэлектронвольтах.

Как видно из формул, зависимости для случаев $\mathrm{H}-\mathrm{W}$, $\mathrm{D}-\mathrm{W}$ и $\mathrm{He}-\mathrm{W}$ весьма похожи и аппроксимируются аналогичными зависимостями.

\section{3. Распределение пробегов по глубине}

В настоящем разделе приводятся результаты, полученные для различных систем с использованием потенциалов с притягивающей ямой. На рис. 5 и 6 приведены распределения пробегов в диапазоне энергий $0.1-100 \mathrm{keV}$ для систем $\mathrm{H}-\mathrm{Si}$ и $\mathrm{D}-\mathrm{W}$ соответственно. В табл. 1 и 2 указаны характеристики данных распределений, которые позволяют построить распределение пробегов с помощью интерполяции, не проводя расчет.

Положение максимума $C$ близко к значению среднего пробега и зависит от асимметрии пика. Как показано на рис. 7, отношение LHW/C и RHW/C с ростом энергии резко уменьшается, пик становится более выраженным. При больших энергиях левая полуширина пика больше правой.

\section{Заключение}

1. Получены распределения пробегов и средние пробеги для атомов $\mathrm{H}, \mathrm{D}, \mathrm{He}$ с энергиями в диапазоне $0.1-100 \mathrm{keV}$ в аморфном W и $\mathrm{Si}$ в случае нормального падения налетающих частиц.

2. Расчеты проводились как в приближении парных соударений, так и более точным методом расчета траекторий частиц. Показано, что результаты обоих методов согласуются между собой и с экспериментальными данными по средним пробегам для системы $\mathrm{H}-\mathrm{Si}$. Для ускорения расчетов пробегов в диапазоне энергий $E=0.1-100 \mathrm{keV}$ мы рекомендуем использовать код, построенный на приближении парных соударений.

3. Оценено влияние наличия притягивающей ямы в потенциале взаимодействия налетающей частицы с атомами твердого тела на результаты расчетов. При использовании потенциалов с ямой (DFT) и без ямы (ZBL) различие пробегов при энергии $100 \mathrm{eV}$ составляет 14,17 и $23 \%$ для систем $\mathrm{H}-\mathrm{W}, \mathrm{D}-\mathrm{W}$ и $\mathrm{He}-\mathrm{W}$ соответственно. С ростом энергии влияние притягивающей ямы ослабевает.

\section{Финансирование работы}

Работа выполнена в рамках государственного задания Министерства образования и науки РФ для Федерального государственного бюджетного учреждения науки Физико-технического института им. А.Ф. Иоффе Российской академии наук.

\section{Конфликт интересов}

Авторы заявляют, что у них нет конфликта интересов.

\section{Список литературы}

[1] Кивилис В.М., Парилис Э.С., Тураев Н.Ю. // ДАН. 1967. T. 173. № 4. C. 805.

[2] Yurasova V.E., Shulga V.I., Karpuzov D.S. // Can. J. Phys. 1968. Vol. 46. N 6. P. 759. DOI: 10.1139/p68-094

[3] Машкова E.C., Молчанов В.А. Применение рассеяния ионов для анализа твердых тел. М.: Энергоатомиздат, 1995. $176 \mathrm{c}$.

[4] Экштайн B. Компьютерное моделирование взаимодействия частиц с поверхностью твердого тела. М.: Мир, 1995. $321 \mathrm{c}$.

[5] Ziegler J.F., Biersack J.P. SRIM - http://www.srim.org

[6] Robinson M.T., Torrens I.M. // Phys. Rev. B. 1974. Vol. 9. N 12. P. 5008. DOI: 10.1103/PhysRevB.9.5008

[7] Бабенко П.Ю., Зиновьев А.Н., Мелузова Д.С., Шергин А.П. // Поверхность. Рентген., синхротр. и нейтрон. исслед. 2018. № 6. С. 7-12. DOI: $10.7868 / \mathrm{S} 0207352818060021$

[Babenko P.Y., Zinoviev A.N., Meluzova D.S., Shergin A.P. // J. Synch. Investig. 2018. Vol. 12. P. 520-525.

DOI: $10.1134 / \mathrm{S} 1027451018030230]$ 
[8] Babenko P.Yu., Deviatkov A.M., Meluzova D.S., Shergin A.P., Zinoviev A.N. // NIMB. 2017. Vol. 406. P. 538-542.

DOI: $10.1016 /$ j.nimb.2016.12.043

[9] Paul H. IAEA. NDS - https://www-nds.iaea.org/stopping

[10] Verlet L. // Phys. Rev. 1967. Vol. 159. N 1. P. 98. DOI: 10.1103/PhysRev.159.98

[11] Ligeon E., Guivarc'h A. // Rad. Eff. 1976. Vol. 27. N 3-4. P. 129. DOI: $10.1080 / 00337577608243025$

[12] Weiser M., Behar M., Kalbitzer S., Oberschachtsiek P., Fink D., Frech G. // NIMB. 1987. Vol. 29. N 3. P. 587. DOI: $10.1016 / 0168-583 X(87) 90073-5$

[13] Zinoviev A.N. // Nucl. Instrum. Methods Phys. Res. B. 2011. Vol. 269. N 9. P. 829. DOI: 10.1016/j.nimb.2010.11.074

[14] Никольский Б.П. Справочник химика. Т. 1. М.: Изд-во Химия, 1966. $337 \mathrm{c.}$

[15] Zinoviev A.N., Norlund K. // Nucl. Instrum. Methods Phys. Res. B. 2017. Vol. 406. P. 511. DOI: $10.1016 /$ j.nimb.2017.03.047

[16] Ziegler J.F., Biersack J.P., Littmark U. // The Stopping and Range of Ions in Solids, The Stopping and Range of Ions in Matter. Vol. 1. NY:: Pergamon, 1985. 321 p. 